\title{
Spatial Stationarity of Ultrawideband and Millimeter Wave Radio Channels
}

\author{
Yi Tan, Jesper Ødum Nielsen, and Gert Frølund Pedersen
}

APNet Section, Department of Electronic Systems, Faculty of Engineering and Science, Aalborg University, 9220 Aalborg, Denmark

Correspondence should be addressed to Yi Tan; junglir@gmail.com

Received 21 September 2015; Revised 10 December 2015; Accepted 31 December 2015

Academic Editor: Christoph F. Mecklenbräuker

Copyright (C) 2016 Yi Tan et al. This is an open access article distributed under the Creative Commons Attribution License, which permits unrestricted use, distribution, and reproduction in any medium, provided the original work is properly cited.

For radio channels with broad bandwidth resource, such as those often used for ultrawideband (UWB) and millimeter wave (mmwave) systems, the Wide-Sense Stationary Uncorrelated Scattering (WSSUS) and spatial stationary assumptions are more critical than typical cellular channels with very limited bandwidth resource. This paper studies spatial stationarity and bandwidth dependency of the Multipath Component (MPC) parameters, and the concept of local region of stationarity (LRS) is used as the measure of the physical stationarity region. LRS calculation results based on channel measurements show that the size of LRS is bandwidth dependent in all measured bands, $2-4 \mathrm{GHz}, 14-16 \mathrm{GHz}$, and $28-30 \mathrm{GHz}$. The results in this paper point out that an inappropriate choice of bandwidth in channel parameter estimation could violate spatial stationary assumptions. The paper indicates LRS sizes for different bandwidths in the three bands.

\section{Introduction}

Wide-Sense Stationary Uncorrelated Scattering (WSSUS) [1] and spatial stationarity (called Homogeneous Channels in [2]) are often the assumed preconditions in the estimation of the Multipath Components (MPCs) of wireless radio channels. Given the wireless channel impulse response in the representation of $h(r, t, \tau)$, then those assumptions limit the wireless channel to be stationary or quasi-stationary in a certain range of space $(r)$, time $(t)$, and delay $(\tau)$. Once any one of the assumptions is violated in the postprocessing of measurement data, the MPC parameter estimation results could be distorted and blurred.

The frequency resource between $3 \mathrm{GHz}$ and $10 \mathrm{GHz}$ is often used for ultrawideband (UWB) systems and is denoted as the Low Band (LB) in this work. Such UWB channels normally have a fractional bandwidth of more than $20 \%$. The MPC parameters in this case are frequency dependent $[3,4]$, and the US assumption is incorrect for the whole bandwidth of such a UWB system. Besides that, the wide bandwidth allows a desirable extremely high spatial resolution of channel sounding on one hand, but, on the other hand, it may become very sensitive to the measurement locations and therefore the spatial stationary assumption could be violated as well.
For the frequency resource between $6 \mathrm{GHz}$ and $60 \mathrm{GHz}$, denoted here as the High Band (HB), it is expected to be used in future millimeter wave (mmwave) systems. For such mmwave system channels, the bandwidth resources are about $7 \mathrm{GHz}$ in the $60 \mathrm{GHz}$ band (unlicensed) and about $1 \mathrm{GHz}$ in the $28 \mathrm{GHz}$ and $38 \mathrm{GHz}$ bands $[5,6]$. Since the fractional bandwidth is about $10 \%$ or less, it could be defined as a narrowband channel and the space-alternating generalized expectation-maximization (SAGE) [7] estimates of some real channel measurements in certain scenarios do show narrowband properties [8]. However, the absolute bandwidth resource of the $\mathrm{HB}$ channels is comparable with that in UWB channels in the LB frequency range. Therefore, it is also very sensitive to the measurement locations and the spatial stationary assumption could be violated in the data postprocessing likewise.

Former studies on the spatial stationarity focus mainly on different frequencies $[9,10]$. The contribution of this paper is the study of the spatial stationarity for different bandwidths, which is based on two channel measurement campaigns. The focus is the size difference of the local region of stationarity (LRS; see Section 2) for different bandwidths assumed in the data postprocessing. Both UWB and mmwave channels within the $\mathrm{LB}$ and $\mathrm{HB}$ frequency ranges are covered. 


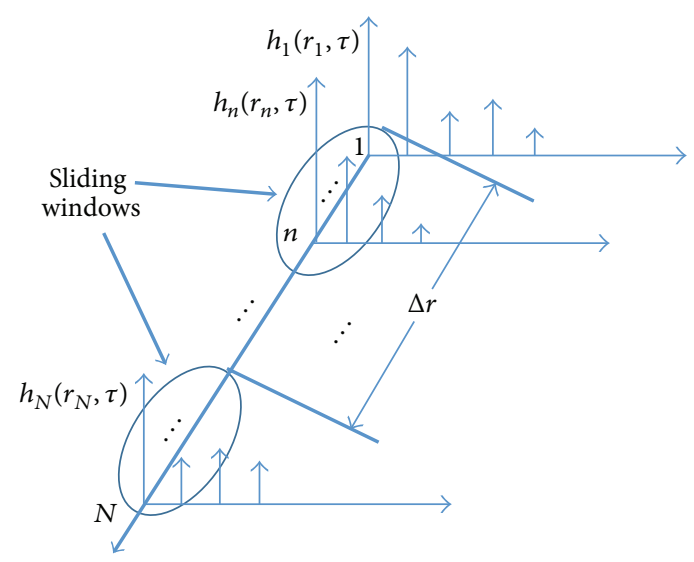

FIGURE 1: Sliding window along antenna array.

Section 2 introduces the method of calculating the LRS. In Section 3, two channel measurement campaigns at Aalborg University are described, and the LRS results are discussed.

\section{Local Region of Stationarity}

There are two approaches to estimating the physical stationarity region: one is based on the Covariance Matrix Distance (CMD) [11-13] and the other is based on the correlation coefficient of the Averaged Power Delay Profiles (APDPs) [14]. The CMD method estimates the correlation matrix and is more suitable in the consideration of MIMO channels and depends on the type of antenna system used in the channel measurement. For the APDP method, this is not the case since it only looks at the PDP and only indirectly at the interantenna correlation. On the other hand, the correlation matrix is a narrowband measure, and hence the wideband properties must be included by averaging over different frequencies (see [12]). The concern of MIMO system is not absolutely necessary in the study of the stationarity, and our study is based on assuming different bandwidths for the data, where some cases are narrowband while some are wideband. Therefore, the most straightforward approach is to use the APDP method for processing the data.

In this paper, we follow both the concept and the definition of the LRS based on APDP in [14]. In the following we assume that the channel is static during the measurements, so that we can describe it as $h(r, \tau)$ without considering the time dimension $t$. For an antenna array consisting of $N$ antennas, the impulse responses acquired by the antennas in the array are $h\left(r_{i}, \tau\right), i=1, \ldots, N$; see Figure 1 .

Here we define $\overline{P_{h}(r, \tau)}$ as the APDP of an $n$-antenna subarray in one sliding window along the antenna array as (1) and define $\overline{P_{h}(r+\Delta r, \tau)}$ as the APDP of another antenna subarray, as it moves along the array with a distance of $\Delta r$. The correlation coefficient of the APDPs is defined as (2), and $c(\Delta r)$ is the correlation coefficient between the APDPs acquired from two antenna subarrays in a distance of $\Delta r$.

$d_{\text {LRS }}$ in (3) determines the stationary interval as the physical stationarity region in which the correlation coefficients between the APDPs, that is, $c(\Delta r)$, are all higher than the threshold $c_{\mathrm{th}}$. We assume the MPC parameters are stationary or quasi-stationary in the spatial dimension inside such physical stationarity region, which we called LRS, and it is used to roughly estimate the size of the physical stationarity region:

$$
\begin{aligned}
\overline{P_{h}(r, \tau)} & =\frac{1}{n} \sum_{i=1}^{n}\left|h\left(r_{i}, \tau\right)\right|^{2}, \\
c(\Delta r) & =\frac{\int \overline{P_{h}(r, \tau)} * \overline{P_{h}(r+\Delta r, \tau)} d \tau}{\left.\max \left\{\int{\overline{P_{h}(r, \tau)}}^{2} d \tau, \overline{P_{h}(r+\Delta r, \tau)}\right)^{2} d \tau\right\}}, \\
d_{\text {LRS }} & =\max \left\{\left.\Delta r\right|_{c(\Delta r)>c_{\text {th }}}\right\} .
\end{aligned}
$$

The value of threshold $c_{\text {th }}$ which determines the size of the LRS is not studied here, because it depends on the channel scenarios and the accuracy requirement of the MPC estimation algorithms used in the data postprocessing, which is beyond the scope of this paper. For convenience of explaining our purpose, we assume $c_{\mathrm{th}}=0.5$ is the threshold in this paper.

Using the larger value of $\int{\overline{P_{h}(r, \tau)}}^{2} d \tau$ and $\int{\overline{P_{h}(r+\Delta r, \tau)}}^{2} d \tau$ in the denominator of (2) is to assure the correlation coefficient is smaller than 1 . And the purpose of using the APDP in the equation is for eliminating fast fading as mentioned in [14].

\section{Local Region of Stationarity for Measured Channels}

Two indoor measurement campaigns were performed in the APNet Section, Department of Electronic Systems, Aalborg University. The indoor scenarios were widely different in these two campaigns. One was in the basement in a big, mostly empty space; the other one was in a filled laboratory. In addition, the antennas used were different as well. The measurements in the basement were made in both LB and $\mathrm{HB}$ frequencies, whereas in the laboratory area only $\mathrm{HB}$ frequencies were used.

3.1. Setup of the Indoor Channel Measurement Campaign in the Basement. A VNA combined with a large virtual Uniform Circular Array (UCA) was used, simultaneously measuring the frequency band of $2 \sim 4 \mathrm{GHz}$ in the $\mathrm{LB}$ and the frequency bands of $14 \sim 16 \mathrm{GHz}$ and $28 \sim 30 \mathrm{GHz}$ in the $\mathrm{HB}$. The volume of the empty space in the basement is $7.85 \mathrm{~m} \times 7.71 \mathrm{~m}$; see Figure 2. Both transmitter $(\mathrm{Tx})$ and receiver $(\mathrm{Rx})$ were using a commercial biconical antenna from A-INFO SZ-2003000/P [15], which has an omnidirectional radiation pattern in the $\mathrm{H}$-plane (horizontal plane) from $2 \mathrm{GHz}$ to $30 \mathrm{GHz}$, and the E-plane (elevation plane) in different frequencies also shows good consistency. Both $\mathrm{Tx}$ and $\mathrm{Rx}$ test stations were fixed at $1 \mathrm{~m}$ height. The Tx was a single fixed antenna. Using a mechanical positioner, the $\mathrm{Rx}$ formed a large virtual UCA consisting of 720 antenna locations around a circle with the radius of $0.5 \mathrm{~m}$. The space between two adjacent virtual 


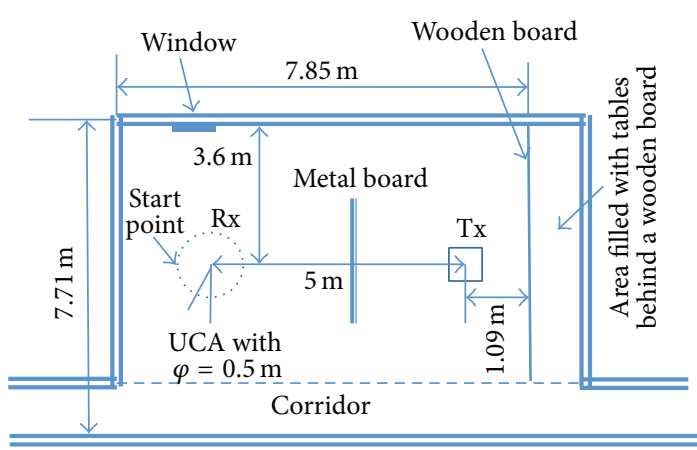

(a)

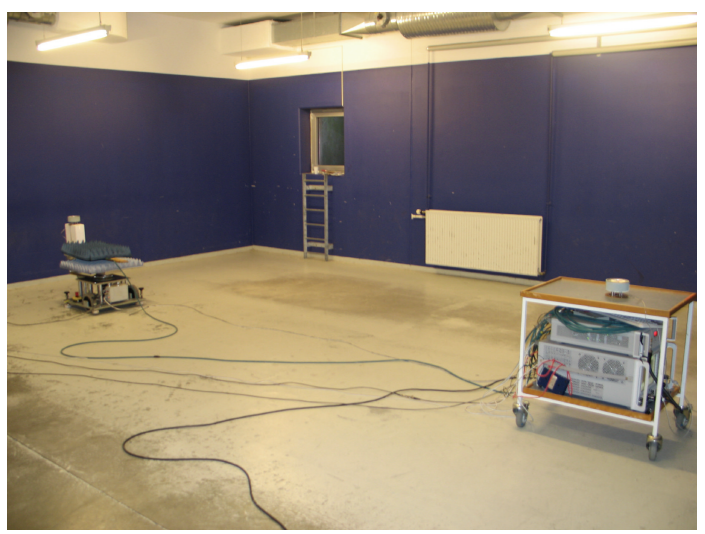

(b)

FIGURE 2: (a) Floorplan of the basement, (b) photo of real channel environment.

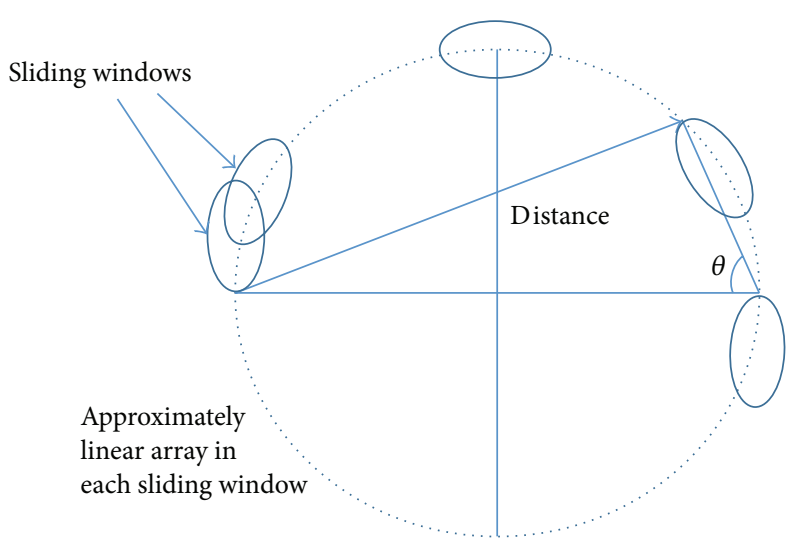

FIGURE 3: Approximation of linear antenna array.

antenna locations was $0.0044 \mathrm{~m}$, less than $\lambda / 2(0.005 \mathrm{~m})$ at $30 \mathrm{GHz}$. The measurement contains both line-of-sight (LOS) and non-LOS (NLOS) scenarios. A metal board was placed between Tx and Rx for the NLOS measurement.

3.2. LRS for $L B$ and $H B$ Channels in the Basement. The calculations are based on 16 consecutive virtual antenna locations in sliding windows along half of the virtual UCA, where each can be seen as an approximately linear antenna subarray; see Figure 3. The distance between two virtual linear antenna arrays was calculated by trigonometry. The physical distance between the two ends of the virtual linear antenna array is less than $0.07 \mathrm{~m}$. We assume that, within this distance, the channel is stationary and the MPC parameters do not change. The far field distance for a single biconical antenna is about $3 \mathrm{~m}$, based on the equation of $2 D^{2} / \lambda$ at $30 \mathrm{GHz}$.

The LRS calculations are based on the measured data filtered for different bandwidths: $2 \mathrm{GHz}, 800 \mathrm{MHz}$, and $200 \mathrm{MHz}$, centered in each of the three measured bands. All the calculations are based on normalized APDPs $\left(\overline{P_{h}(r, \tau)}\right.$ and $\overline{P_{h}(r+\Delta r, \tau)}$ have the same power). The maximum difference in total power between $\int{\overline{P_{h}(r, \tau)}}^{2} d \tau$ and $\int{\overline{P_{h}(r+\Delta r, \tau)}}^{2} d \tau$ in different sliding windows is about $10 \mathrm{~dB}$, for both LOS and NLOS scenarios.

The correlation coefficient $c(\Delta r)$ of the APDPs obtained for different frequency bands is shown in Figure 4 . The size of LRSs in the $2 \mathrm{GHz}$ bandwidth case is clearly different from the LRSs in the $200 \mathrm{MHz}$ and $800 \mathrm{MHz}$ bandwidth cases, and, generally, the broader the bandwidth, the smaller the size of the LRS.

3.3. Setup of the Indoor Channel Measurement Campaign in the Laboratory. A VNA combined with a large virtual Uniform Linear Array (ULA) channel sounding system was used in the $\mathrm{HB}$ frequency range of $27.5-32.5 \mathrm{GHz}$. The volume of the laboratory is $8.75 \mathrm{~m}$ times $5.7 \mathrm{~m}$, filled with three test benches and two large cupboards; see Figure 5. The transmitter $(\mathrm{Tx})$ was a $\lambda / 4$ single monopole omnidirectional antenna in the middle of a $10 \mathrm{~cm} \times 10 \mathrm{~cm}$ ground plane, fixed at the height of $126 \mathrm{~cm}$. Radiation patterns of this simple and widely used structure are shown in Figure 6. The receiver $(\mathrm{Rx})$ used the same type of monopole antenna mounted on a linear sledge, which was moved to form a 400 element large virtual ULA $(1 \mathrm{~m})$; see the colored lines in Figure 5(a). The space between adjacent virtual antennas was $2.5 \mathrm{~mm}(\lambda / 4$ at $30 \mathrm{GHz}$ ), and the height of whole antenna array was $107 \mathrm{~cm}$. The Tx position P4 was for the LOS measurement, with corresponding Rx positions at P1 $(\mathrm{V}+\mathrm{H}), \mathrm{P} 2(\mathrm{~V})$, and $\mathrm{P} 3(\mathrm{~V})$ in black color; Tx at $\mathrm{P} 5$ position was for NLOS measurements, with corresponding $\mathrm{Rx}$ positions at $\mathrm{P} 1(\mathrm{~V}+\mathrm{H}), \mathrm{P} 6(\mathrm{~V}+\mathrm{H})$, and $\mathrm{P} 7 \mathrm{H}$ ) in red color (LOS and NLOS measurement both performed at $\mathrm{P} 1$ position).

3.4. LRS for the HB Channels in the Laboratory. $\mathrm{P} 4$ versus $\mathrm{P} 1(\mathrm{~V}) / \mathrm{P} 2(\mathrm{~V}) / \mathrm{P} 3(\mathrm{~V})$ and P5 versus P1 (V)/P6 (V) could be seen as scenarios with the Rx gradually moving away from the Tx. The APDPs are based on 16 consecutive virtual antenna locations as sliding window along the $1 \times 400$ virtual ULA; refer to Figure 1 . The physical distance between the two ends of the virtual linear antenna array is $0.1375 \mathrm{~m}(15 \times \lambda / 4+2 \times$ $50 \mathrm{~mm}$ ), and we assume that, within this distance, the channel is stationary and the MPC parameters do not change. The far 


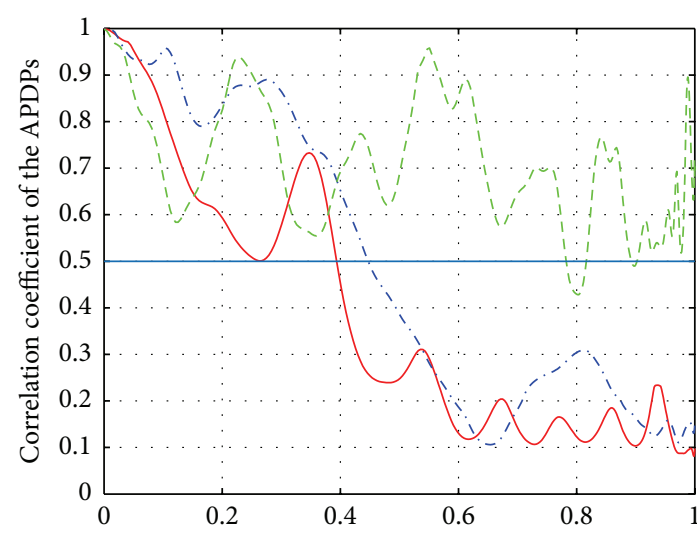

Physical distance between the antenna subarrays $(\mathrm{m})$

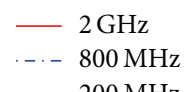

$200 \mathrm{MHz}$

(a)

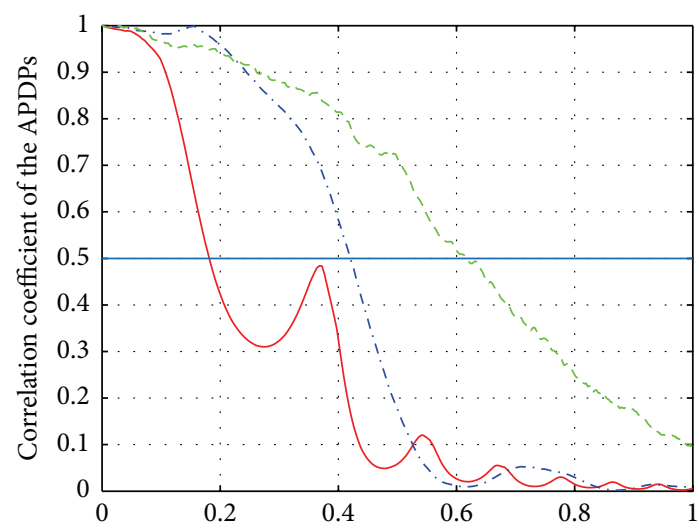

Physical distance between the antenna subarrays $(\mathrm{m})$

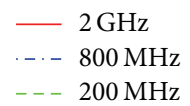

(c)

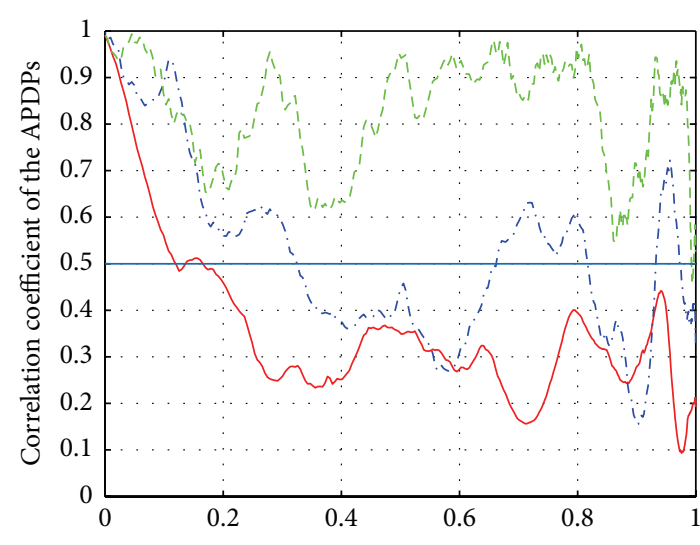

Physical distance between the antenna subarrays $(\mathrm{m})$

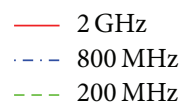

- - $200 \mathrm{MHz}$

(e)

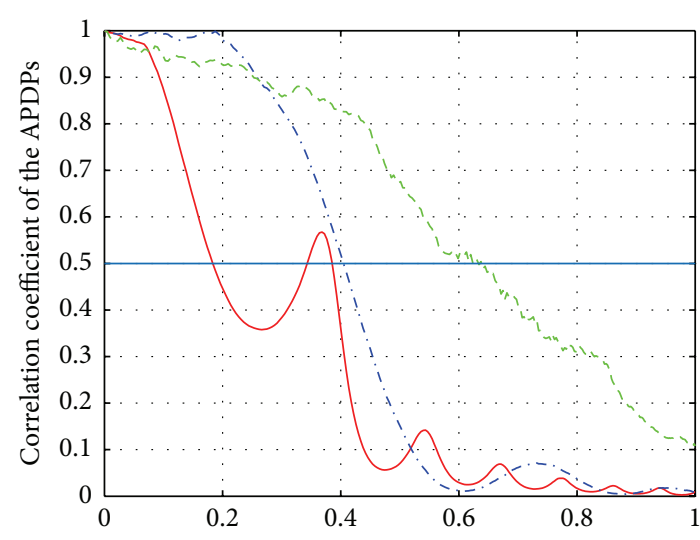

Physical distance between the antenna subarrays $(\mathrm{m})$

$-2 \mathrm{GHz}$

-.. $800 \mathrm{MHz}$

$200 \mathrm{MHz}$

(b)

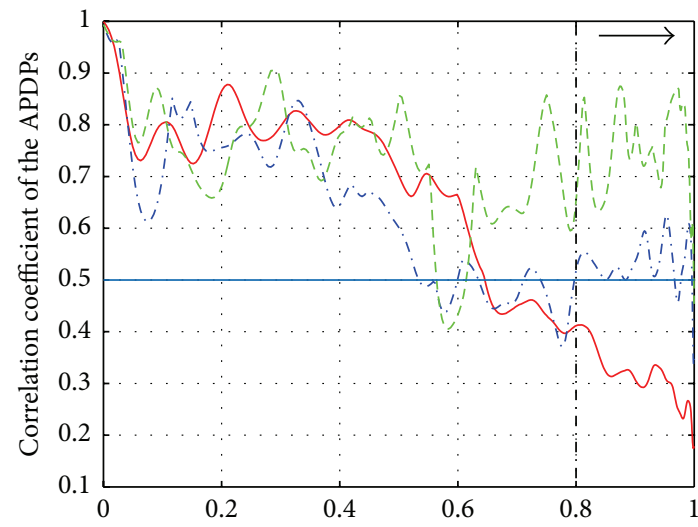

Physical distance between the antenna subarrays $(\mathrm{m})$

$-2 \mathrm{GHz}$

...- $800 \mathrm{MHz}$

$---200 \mathrm{MHz}$

(d)

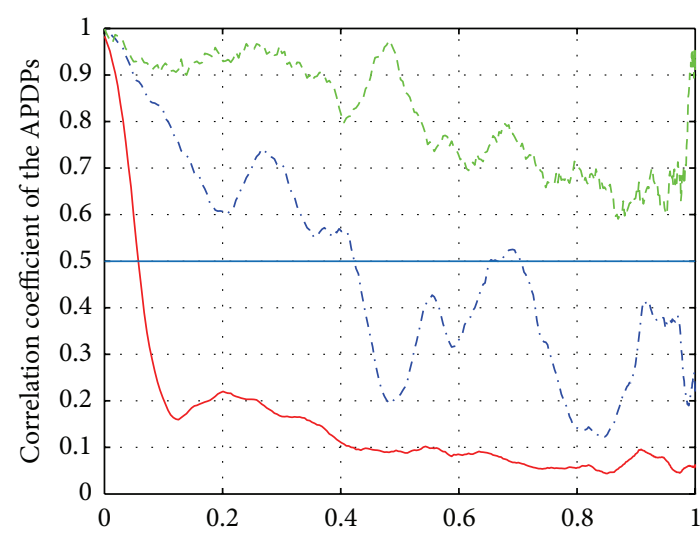

Physical distance between the antenna subarrays $(\mathrm{m})$

$-2 \mathrm{GHz}$

... $800 \mathrm{MHz}$

- - $200 \mathrm{MHz}$

(f)

Figure 4: Correlation coefficient of the APDPs for the channel measurement in the basement: LOS scenarios: (a) 2-4 GHz in LB, (b) 14$16 \mathrm{GHz}$ in $\mathrm{HB}$, and (c) $28-30 \mathrm{GHz}$ in $\mathrm{HB}$; NLOS scenario: (d) $2-4 \mathrm{GHz}$ in $\mathrm{LB}$, (e) $14-16 \mathrm{GHz}$ in $\mathrm{HB}$, and (f) $28-30 \mathrm{GHz}$ in $\mathrm{HB}$. 


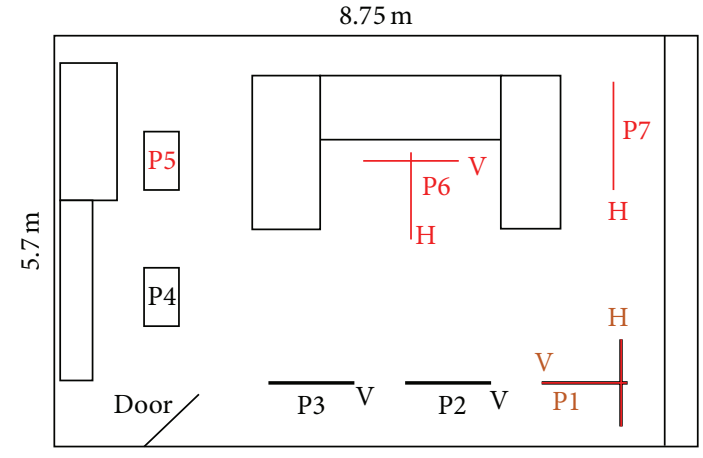

(a)

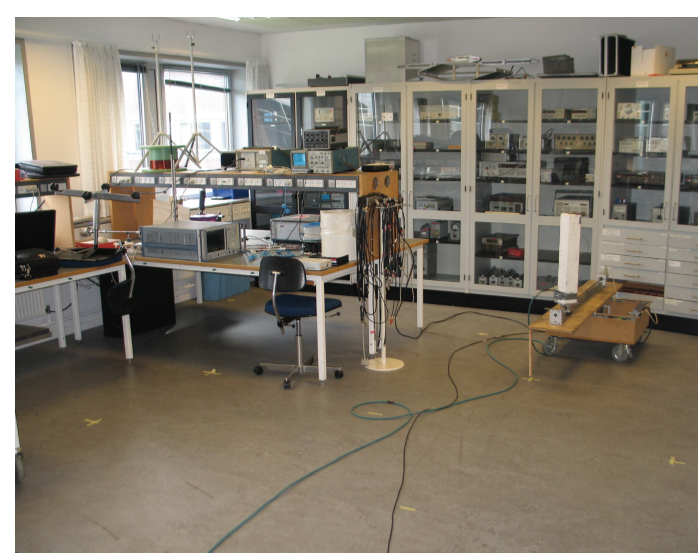

(b)

FIGURE 5: (a) Floorplan of the laboratory, (b) photo of real channel environment (Rx consists of ULA).

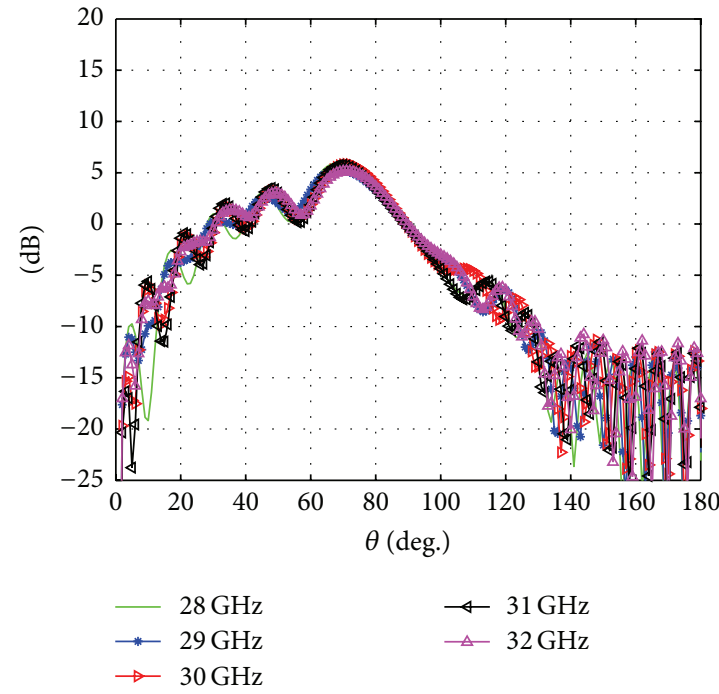

(a)

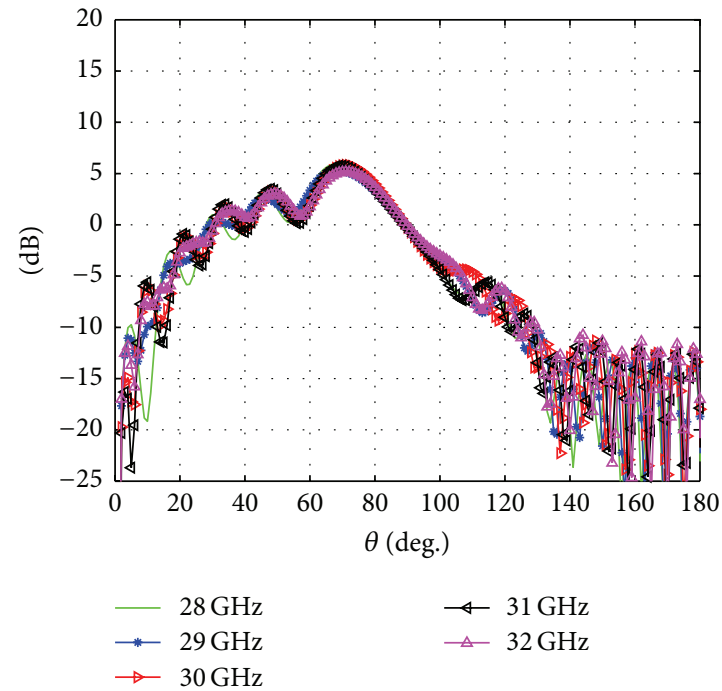

(b)

FIGURE 6: Omnidirectional monopole antenna, frequency points: $28 \mathrm{GHz}$ to $32 \mathrm{GHz}$ with $1 \mathrm{GHz}$ step. (a) The absolute gain on the plane of $\phi=0$ in spherical coordination system, (b) the absolute gain on the plane of $\phi=90$.

field of a single monopole antenna is about $4 \mathrm{~m}$, based on the equation of $2 D^{2} / \lambda$ at $30 \mathrm{GHz}$.

As above, the LRS calculations are based on the data filtered for bandwidths of $2 \mathrm{GHz}, 800 \mathrm{MHz}$, and $200 \mathrm{MHz}$, each centered in the frequency band. The APDPs were normalized, and the maximum difference in total power between $\int{\overline{P_{h}(r, \tau)}}^{2} d \tau$ and $\int{\overline{P_{h}(r+\Delta r, \tau)}}^{2} d \tau$ in different sliding windows is about $23 \mathrm{~dB}$, for both LOS and NLOS scenarios.

The LRS results are very similar to those in Figure 4; the LRS of P4 versus $\mathrm{P} 3$ for LOS scenario and the LRS of P5 versus P6 for NLOS scenario are shown in Figure 7. The size of LRS is bandwidth dependent as expected; in general the broader the bandwidth, the smaller the size of LRS, and the results of the correlation coefficient of the APDPs have the same trend for both LOS and NLOS measurements.
3.5. Discussion. From Figures 4 and 7, a general observation is that a wider bandwidth leads to a smaller LRS for a given threshold of correlation coefficient of the APDPs. However, in Figure 4 this is clearest for the $28-30 \mathrm{GHz}$ band and the LOS case and less clear for the lower bands and the NLOS case. An explanation could be that the path loss tends to increase with frequency, so that non-LOS paths are relatively more important for lower bands than for higher bands. Using a larger UCA (larger physical distance of two approximately linear antenna arrays) could possibly lead to a clearer trend for the lower bands. Notice that we can start to observe the same trend for physical distances larger than about $0.8 \mathrm{~m}$ in Figure 4(d).

Different choices of sounding bandwidths imply different spatial resolutions. For the three bandwidths used here the spatial resolutions are about $0.15 \mathrm{~m}, 0.375 \mathrm{~m}$, and $1.5 \mathrm{~m}$ for 


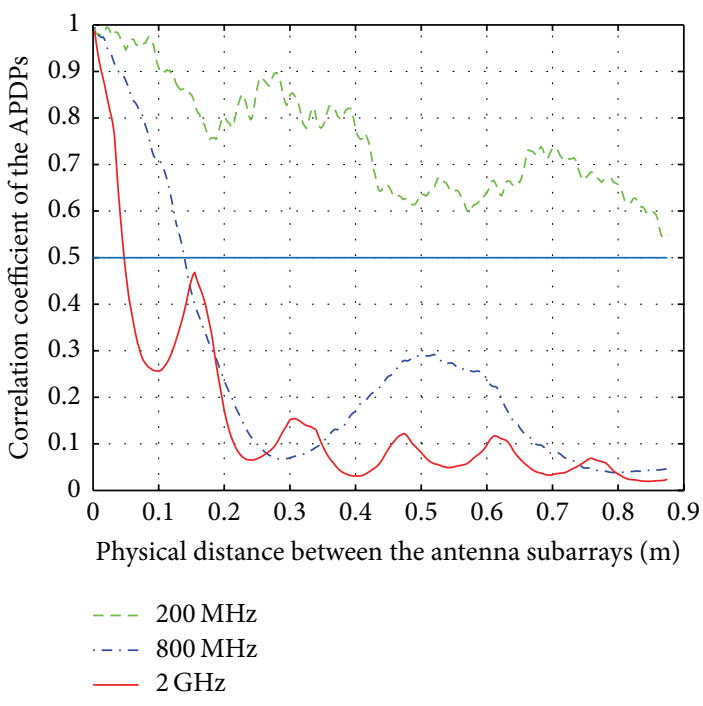

(a)

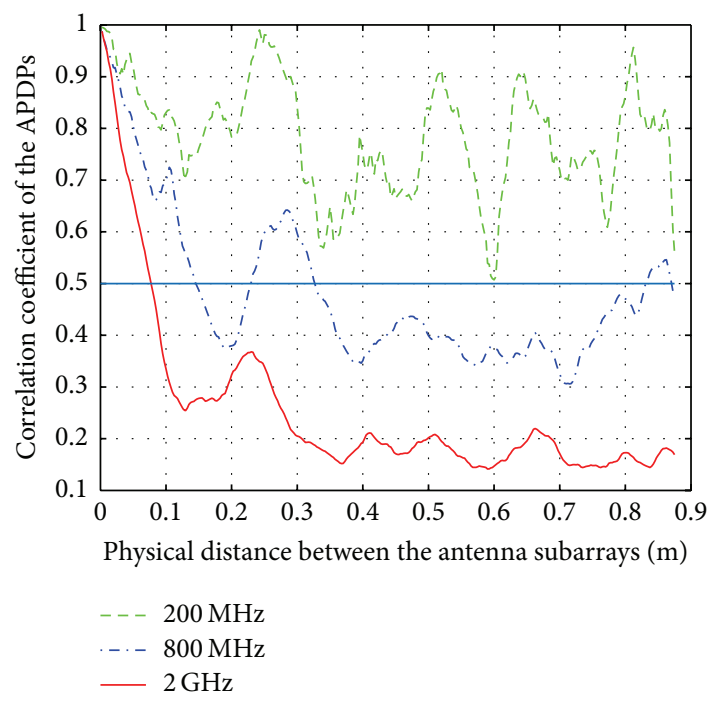

(b)

FIGURE 7: Correlation coefficient of the APDPs at different bandwidth for the channel measurement in the laboratory: (a) LOS scenario: P4 versus P3; (b) NLOS scenario: P5 versus P6.

the $2 \mathrm{GHz}, 800 \mathrm{MHz}$, and $200 \mathrm{MHz}$ bandwidths, respectively. Observing the curves in the $14-16 \mathrm{GHz}$ and $28-30 \mathrm{GHz}$ bands for the LOS case in Figure 4 and the curves for LOS scenario in Figure 7, the size of the LRS results is proportional to spatial resolutions corresponding to the bandwidth of the channel sounding signals. However, for the $2-4 \mathrm{GHz}$ band and the NLOS scenarios, the situation is proportional as well but with more deviations.

Previously published results have shown a reduced spatial correlation with increasing carrier frequency, measured with fixed physical distance between the Tx and $\mathrm{Rx}[9,10]$. This means the coherence distance is becoming smaller as the frequency goes higher. A similar conclusion can be drawn from Figure 4 for the NLOS cases. However, for the LOS cases it seems that the LRS sizes are almost independent of the frequency, which could be due to the main received power being concentrated in the LOS path and therefore the channel does not depend as much on physical dimensions of the environment, compared to the NLOS case.

\section{Conclusion}

For characterizing channels used for UWB and mmwave communication systems, channel sounding with very high spatial resolution may be used. Estimating channel parameters from such data often relies on stationarity assumptions; therefore the size of the so-called local region of stationarity (LRS) is critical. This paper applies the LRS to two sets of indoor channel measurements which includes the frequency band of $2 \sim 4 \mathrm{GHz}$, typically used for UWB, and the frequency bands of $14 \sim 16 \mathrm{GHz}$ and $28 \sim 30 \mathrm{GHz}$ typically used for mmwave systems. The results show that the size of the LRS is bandwidth dependent for both types of channels. For the LOS channels the LRS is largely independent of the frequency band, whereas, for the NLOS channels this is not the case.
The LRS should be considered carefully when performing parameter estimation in wideband channels, such as using the DoA/ToA algorithms of beamforming, Capon, MUSIC, ESPRIT, JADE [16], SAGE, and so forth. An inappropriate choice of bandwidth could violate the spatial stationary assumption, and the estimation results could be blurred and distorted.

\section{Conflict of Interests}

The authors declare that there is no conflict of interests regarding the publication of this paper.

\section{Acknowledgments}

The authors would like to thank Assistant Professor Wei Fan, Kim Olesen, and Kristian Bank for their invaluable work in the channel measurement campaigns. Thanks are also due to the anonymous reviewers for their insightful technical comments.

\section{References}

[1] P. Bello, "Characterization of randomly time-variant linear channels," IEEE Transactions on Communications System, vol. 11, no. 4, pp. 360-393, 1963.

[2] A. Paulraj, R. Nabar, and D. Gore, Introduction to Space-Time Wireless Communications, chapter 2, Cambridge University Press, Cambridge, UK, 1st edition, 2008.

[3] A. F. Molisch, "Ultra wideband channels," in Wireless Communications, chapter 6.6 , p. 118, John Wiley \& Sons, 2nd edition, 2010.

[4] A. F. Molisch, "Ultra-wide-band propagation channels," Proceedings of the IEEE, vol. 97, no. 2, pp. 353-371, 2009. 
[5] A. F. Molisch and F. Tufvesson, "Propagation channel models for next-generation wireless communications systems," IEICE Transactions on Communications, vol. E97-B, no. 10, pp. 20222034, 2014.

[6] S. K. Yong and C.-C. Chong, "An overview of multigigabit wireless through millimeter wave technology: potentials and technical challenges," EURASIP Journal on Wireless Communications and Networking, vol. 2007, Article ID 078907, 2007.

[7] B. H. Fleury, M. Tschudin, R. Heddergott, D. Dahlhaus, and K. I. Pedersen, "Channel parameter estimation in mobile radio environments using the SAGE algorithm," IEEE Journal on Selected Areas in Communications, vol. 17, no. 3, pp. 434-450, 1999.

[8] K. Haneda, C. Gustafson, and S. Wyne, "60 GHz spatial radio transmission: multiplexing or beamforming?" IEEE Transactions on Antennas and Propagation, vol. 61, no. 11, pp. 5735-5743, 2013.

[9] J. Liu, B. Allen, W. Q. Malik, and D. J. Edwards, "A measurement based spatial correlation analysis for MB-OFDM ultra wideband transmissions," in Proceedings of the LAPC Conference, Loughborough, UK, April 2005.

[10] Y. Wang, I. Kovacs, G. F. Pedersen, and K. Olesen, "Spatial correlation of PAN UWB-MIMO channel including user dynamics," COST 2100 TD(07), Deparment of Electronic Systems, Aalborg University, Duisburg, Germany, 2007.

[11] M. Herdin and E. Bonek, "A MIMO Correlation Matrix based Metric for Characterizing Non-Stationarity," in Proceedings of the 13th IST Mobile \& Wireless Communications Summit, Talk: IST Mobile \& Wireless Communications, p. 5, Lyon, France, 2004.

[12] M. Herdin, N. Czink, H. Ozcelik, and E. Bonek, "Correlation matrix distance, a meaningful measure for evaluation of nonstationary MIMO channels," in Proceedings of the 61st IEEE Vehicular Technology Conference (VTC '05), vol. 1, pp. 136-140, IEEE, Stockholm, Sweden, May-June 2005.

[13] O. Renaudin, V.-M. Kolmonen, P. Vainikainen, and C. Oestges, "Impact of correlation matrix estimation accuracy on the computation of stationarity intervals," in Proceedings of the 4th European Conference on Antennas and Propagation (EuCAP'10), pp. 1-5, Barcelona, Spain, April 2010.

[14] A. Gehring, M. Steinbauer, I. Gaspard, and M. Grigat, "Empirical channel stationarity in urban environments," in Proceedings of the European Personal Mobile Communications Conference (EPMCC '01), Vienna, Austria, February 2001.

[15] Chengdu A-INFO Inc, http://www.ainfoinc.com/en/index.asp.

[16] M. C. Vanderveen, A.-J. van der Veen, and A. Paulraj, "Estimation of multipath parameters in wireless communications," IEEE Transactions on Signal Processing, vol. 46, no. 3, pp. 682690, 1998. 


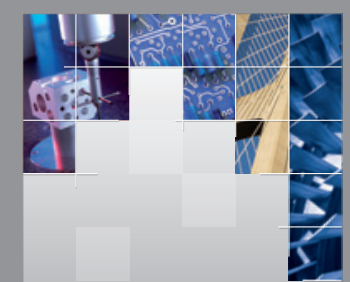

\section{Enfincering}
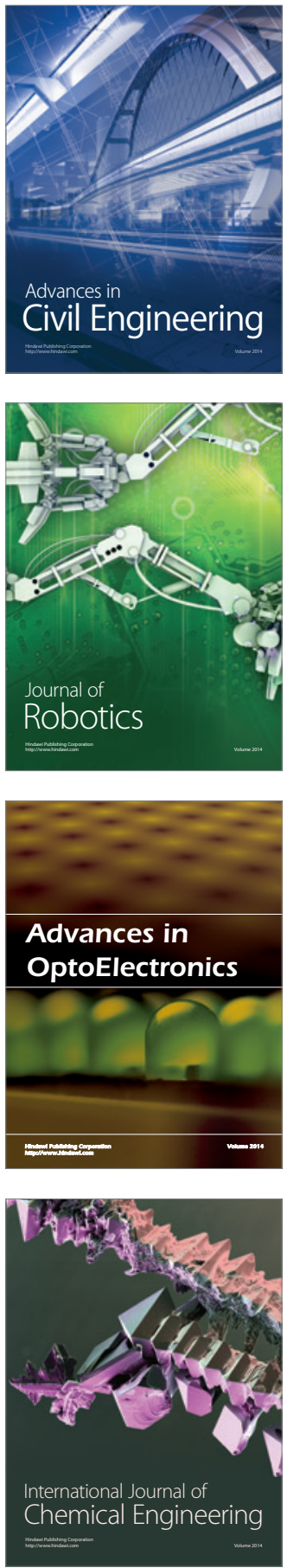

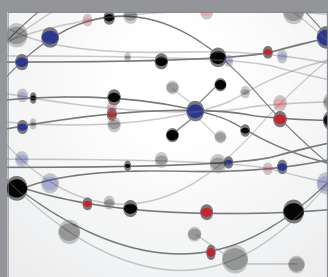

The Scientific World Journal

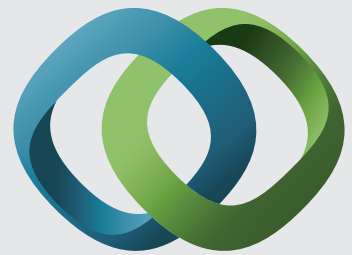

\section{Hindawi}

Submit your manuscripts at

http://www.hindawi.com
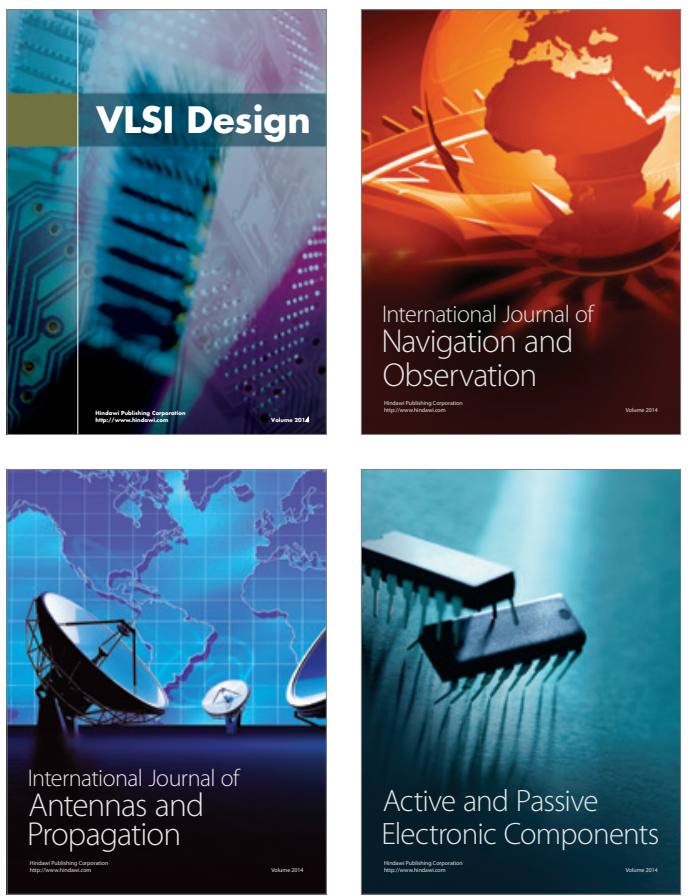
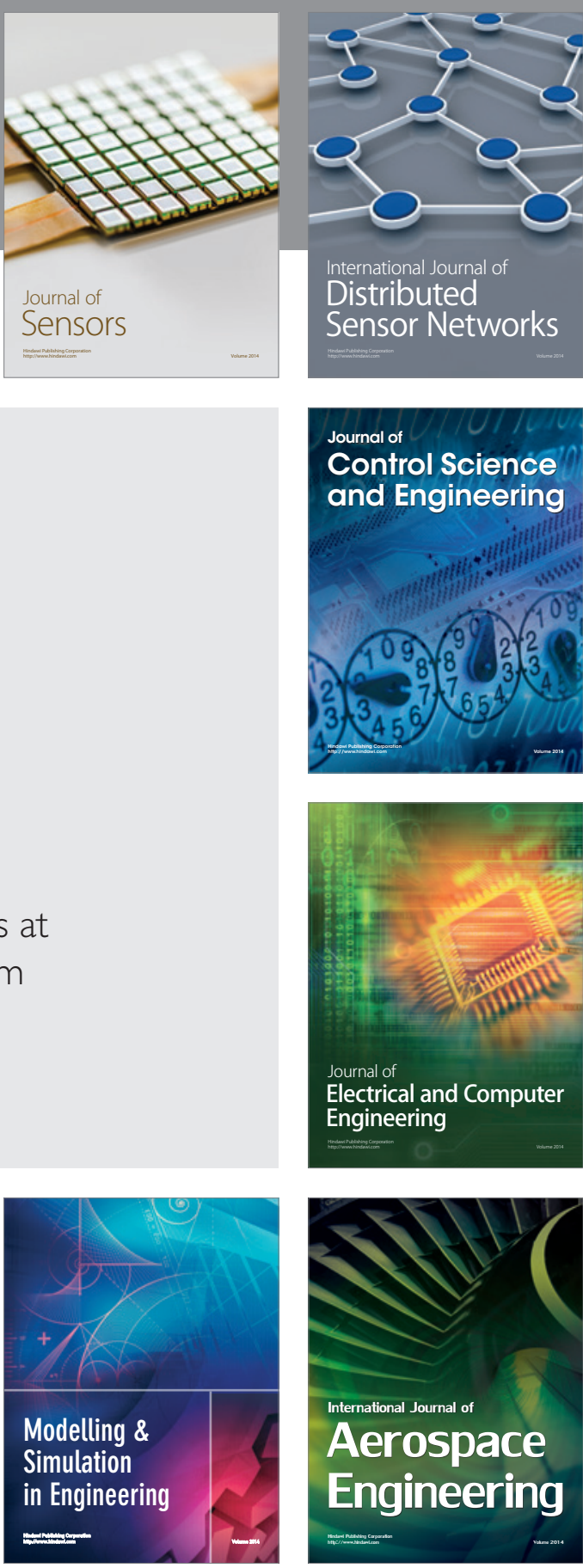

International Journal of

Distributed

Sensor Networks

Journal of

Control Science

and Engineering
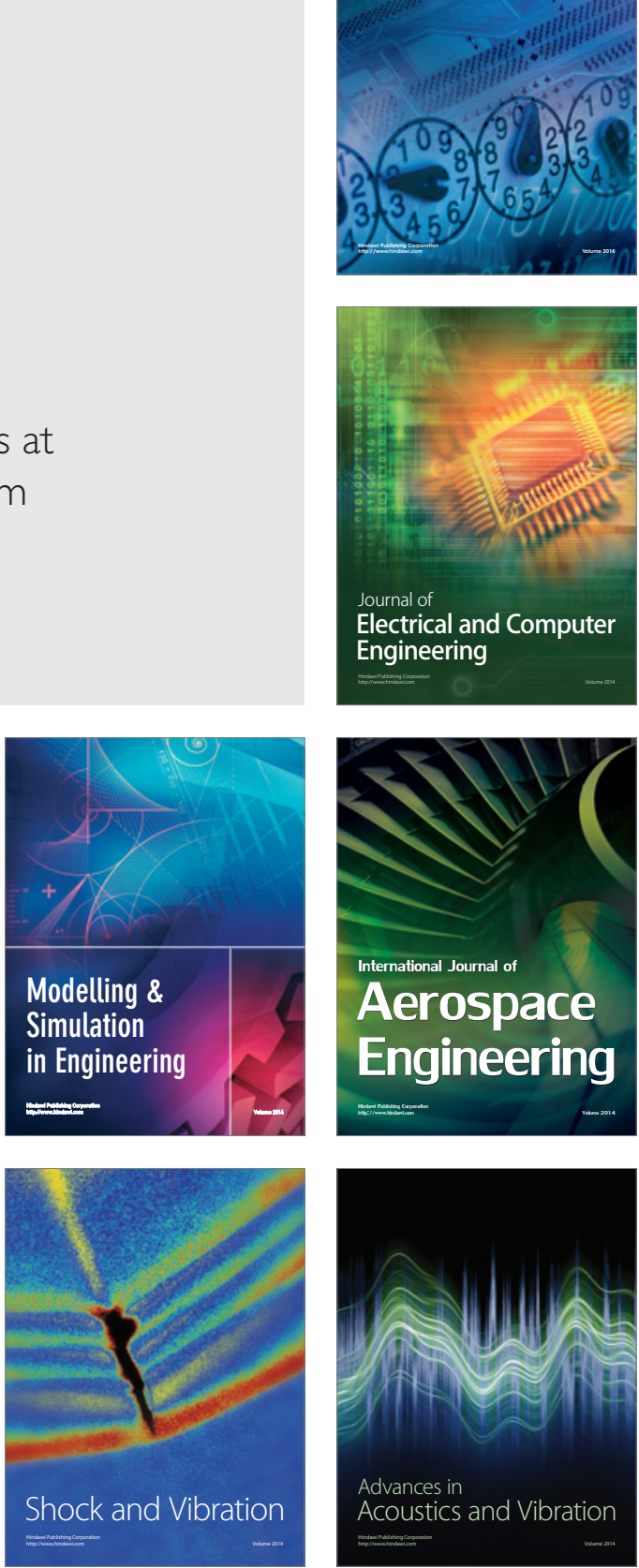